\title{
A simulating cognitive system with adaptive capability
}

\author{
Hans H. Diebner* \\ Center for Art and Media, Institute for Basic Research, Lorenzstr. 19, 76135 Karlsruhe, Germany \\ Received 6 March 2001; received in revised form 12 July 2001; accepted 18 July 2001
}

\begin{abstract}
Dedicated to the memory of Michael Conrad, this paper builds on his seminal ideas expressed in his famous book Adaptability, as well as in his later works. We investigate a recently published adaptive system for the instantaneous recognition of dynamics with respect to its adaptability to the Lorenz system. The system consists of a pool of internal dynamical elements. These elements are defined through a set of parameter values that encode for a specific dynamics behavior. If the system is now faced with an unknown external dynamics - unknown with respect to the parameterit is capable not only to recognize the dynamics but also to adapt to the correct dynamics, which in turn leads to a simulation capability. The system impressively quickly follows the sudden qualitative changes of the external dynamics. The adaptation works even quicker when the correct dynamics are already represented within the internal pool. This leads to the idea of memorizing the represented dynamics within the pool, whereby the elements that correspond to rarely externally presented dynamics can be given free for the adaptation and memorization of more frequently presented dynamics. (C) 2002 Elsevier Science Ireland Ltd. All rights reserved.
\end{abstract}

Keywords: Adaptability; External dynamics; Internal dynamics; Lorenz system

\section{Introduction}

The adaptive simulating system presented in this paper, in memory of Michael Conrad, is a tiny step towards the goal he formulated:

The goal is to understand the dynamics of natural evolutionary systems and to use the principles to design computing systems with adaptive capabilities.
* Fax: + 49-721-8100-1139.

E-mail address: hans@diebner.de (H.H. Diebner).
This formulation can be found on Michael's website but has been stated much earlier in a similar way, for example in his famous book 'Adaptability: The significance of variability from molecule to ecosystem' (Conrad, 1983). Needless to say, Michael's contributions for cognitive systems research were and remain invaluable.

Most impressive for me was Michael's way of thinking in terms of 'first principles'. Guided by this idea, we showed in a previous work that a specific chaos control mechanism introduced by Pyragas (Pyragas, 1992) can be understood as a diffusive process (Hoff et al., 1995), which in turn allows 'implementation' on a molecular level. It has been made evident that chaos plays an impor- 
tant role in understanding brain dynamics (Skarda and Freeman, 1987; Nicolis and Tsuda, 1999; Tsuda, 2001). Additionally, the chaos control mechanism is a candidate for the description of the cognitive processes within the brain (Babloyantz and Lourenço, 1994; Lourenço and Babloyantz, 1994; Hoff, 1994). Therefore, it is highly plausible to use the easily implementable chaos control mechanism for the construction of artificial cognitive systems that are intended to mimic natural cognitive processes. We briefly recapitulate the essential idea of Pyragas' control in the subsequent section.

Furthermore, we are guided by the idea that the brain is not only a perceptive apparatus but also has the capability to simulate (stimulus and simulus) (Rössler, 1974; Prehn, 2001; Nicolis and Tsuda, 1999). We propose an adaptive network for the recognition of measured external dynamics. Thereby, an internal representation of the dynamics is created that is able to simulate and thus has predictive potential very much like our estimating whether or not we can safely cross the street when a car approaches. However, quite frequently we have to react to sudden changes in external dynamics. The alternation between 'stimulus' and 'simulus' leads to a continuous update of the internal representation of the dynamics, which in turn can be used for simulation.

Fig. 1 shows schematically how the proposed system works. To the left, we have an external

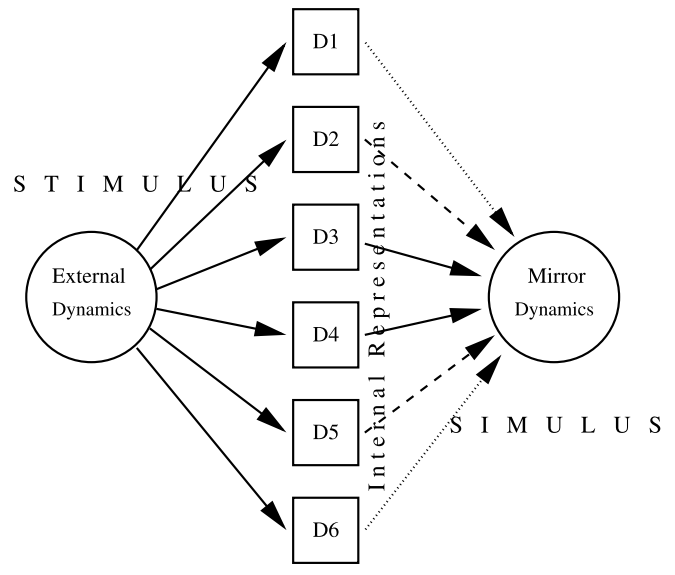

Fig. 1. Scheme of the adaptive system. dynamics (the stimulus), which is 'perceived' by the system. To the right, we see the internal representation of the external dynamics as a 'mirror'. These dynamics are the simulative part of the system (simulus). The kernel of the system is a pool of dynamical modules, each of which is controlled by the external dynamics. The strength of the control term, which is explained in detail in the following section, serves as a measure of 'fitness' of the corresponding module. The parameters of the modules are used in a superpositional manner, weighted by their 'fitness', to build up the parameter of the simulus. In the scheme depicted in Fig. 1, the modules $D_{3}$ and $D_{4}$, for example, fit best to the stimulus and, therefore, contribute with a larger weight (bold arrows) to the construction of the simulus. $D_{1}$ and $D_{6}$, for example, fit worse and contribute less (dashed arrows) to build up the simulus.

A first version using an external signal computed from the Rössler system (Rössler, 1976) has been already published (Diebner et al., 2001), which we recapitulate briefly in the following. Thereafter, we show that the adaptation also works with the Lorenz system (Lorenz, 1963), which is nontrivial because the Lorenz system is usually more difficult to treat than the Rössler system, due to its sudden alternation between the two 'wings'.

\section{Brief recapitulation of pyragas' control method}

Assume $\mathbf{x}$ and $\mathbf{x}^{\prime}$ to be the states of two dynamical systems of the same dimension $n$ and the same dynamics $\mathbf{f}$, given by the differential equations:

$$
\begin{aligned}
& \dot{\mathbf{x}}=\mathbf{f}(\mathbf{x}, \beta) ; \quad \beta=\left(\beta_{1}, \beta_{2}, \ldots, \beta_{m}\right) \\
& \dot{\mathbf{x}}^{\prime}=\mathbf{f}\left(\mathbf{x}^{\prime}, \beta^{\prime}\right) ; \quad \beta^{\prime}=\left(\beta_{1}^{\prime}, \beta_{2}^{\prime}, \ldots, \beta_{m}^{\prime}\right)
\end{aligned}
$$

where $\beta$ and $\beta^{\prime}$ are sets of fixed parameters. Assume further that one of the parameters of the unprimed system is different from the corresponding one in the primed system, $\beta_{k} \neq \beta_{k}^{\prime}$, and the others are equal $\beta_{i}=\beta_{i}^{\prime}, i \neq k$. If the difference of at least one pair of corresponding variables (say the first) multiplied by a suitable chosen factor $K$ is added to the unprimed system, given by: 


$$
\begin{gathered}
\dot{x}_{1}=f_{1}\left(x_{1}, x_{2}, \ldots, x_{n} ; \beta\right)+K\left(x_{1}^{\prime}-x_{1}\right) \\
\dot{x}_{2}=f_{2}\left(x_{1}, x_{2}, \ldots, x_{n} ; \beta\right) \\
\vdots \\
\dot{x}_{n}=f_{n}\left(x_{1}, x_{2}, \ldots, x_{n} ; \beta\right)
\end{gathered}
$$

then this unprimed system will be forced to the dynamics of the primed controlling system, at least if the difference of the dynamics is not too extreme. If the difference of the system parameters is relatively small, the value of the control term $K\left(x_{1}^{\prime}-x_{1}\right)$ will be negligible in the long term. The usual application of Pyragas' method is to stabilize previously unstable periodic orbits in nonlinear systems in the chaotic regime (Hoff, 1997). In the following we deviate slightly from this original application by using Pyragas' control method for synchronization of two dynamical systems and refraining from being able to stabilize with an almost vanishing control term. We instead use the absolute values of the control terms of Pyragas' force control method to compare different dynamical modules as depicted in Fig. 1 and as described in detail in the sequel.

\section{Introduction of the adaptive system}

In a first approach we use the time series of the $x$-variable of Rössler's system (Rössler, 1976)

$\dot{x}_{\mathrm{E}}^{\mathrm{R}}=-y_{\mathrm{E}}^{\mathrm{R}}-z_{\mathrm{E}}^{\mathrm{R}}$

$\dot{y}_{\mathrm{E}}^{\mathrm{R}}=x_{\mathrm{E}}^{\mathrm{R}}+0.2 y_{\mathrm{E}}^{\mathrm{R}}$

$\dot{z}_{\mathrm{E}}^{\mathrm{R}}=0.2+x_{\mathrm{E}}^{\mathrm{R}} z_{\mathrm{E}}^{\mathrm{R}}-\alpha_{\mathrm{E}} z_{\mathrm{E}}^{\mathrm{R}}$

as a given external signal. The subscript $\mathrm{E}$ refers to 'external'. The parameter $\alpha_{\mathrm{E}}$ is fixed at 5.7 in the first step, which leads to a chaotic attractor. The superscript $\mathrm{R}$ encodes for 'Rössler' because we want to distinguish it from the later-used Lorenz system.

Now we choose six further Rössler systems with parameters:

$\alpha_{1}=5.64, \quad \alpha_{2}=5.66, \quad \alpha_{3}=5.68$,

$\alpha_{4}=5.72, \quad \alpha_{5}=5.74, \quad \alpha_{6}=5.76$

to constitute a pool of internally given dynamical types. Each of these internal dynamics is forced to the external time series by means of

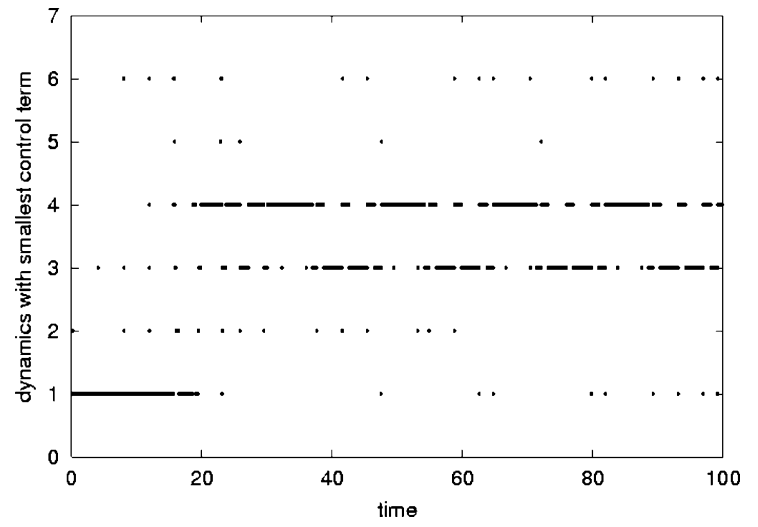

Fig. 2. Switched-on dynamics by time. After a transient phase, the internal representation of the external dynamics switches between two dynamics that are closest to the external one in terms of the difference of the parameter values.

$$
\begin{aligned}
& \dot{x}_{i}^{\mathrm{R}}=-y_{i}^{\mathrm{R}}-z_{i}^{\mathrm{R}}+K\left(x_{\mathrm{E}}^{\mathrm{R}}-x_{i}^{\mathrm{R}}\right) \\
& \dot{y}_{i}^{\mathrm{R}}=x_{i}^{\mathrm{R}}+0.2 y_{i}^{\mathrm{R}} \\
& \dot{z}_{i}^{\mathrm{R}}=0.2+x_{i}^{\mathrm{R}} z_{i}^{\mathrm{R}}-\alpha_{i} z_{i}^{\mathrm{R}} ; \quad i=1,2, \ldots, 6
\end{aligned}
$$

where $K$ is a coupling constant that is chosen to be 1 in the following. At each time step $(h=0.01$ throughout the paper) of the integration of the differential equation we compute the minimum of the absolute value of the six coupling terms. Fig. 2 shows the index (corresponding to the subscripts used in Eq. (4)) of the dynamics that has the smallest control term versus time. The initial conditions of system 1 have been chosen equal to the initial conditions of the external system, which explains that system 1 has the smallest control term in the beginning. In addition, the external system itself has a transient phase in the beginning of about 20 time units, which roughly corresponds to four cycles of the chaotic oscillator. However, after that transient time of $20 \mathrm{U}$, the smallest control term alternates between systems 3 and 4, which is plausible since the parameter value of the external system lies in between the parameter values of that pair of internal systems. Fluctuations cause a negligible number of events where the minima are found in the other systems. These rare events can be suppressed easily by using a moving average of the absolute value of the forcing term over, say, three time steps. Since we here 
focus on the basic properties of the adaptive system we skip this fine-tuning.

One can use the 'switched-on' dynamics, i.e. with the temporarily smallest control term, to reconstruct the attractor of the external Rössler system by using the temporarily produced trajectory pieces, for example. However, since our goal is to construct an adaptive system with simulation capability, we instead create a new element within the internal pool of dynamics that mimics the external dynamics as precisely as possible.

\section{Creation of a mirror dynamics}

To create a new element within the pool of internal dynamics as a mirror of the external dynamics we use the relative frequency $p_{i}$ of the switched-on dynamics to compute a continuous update of the parameter value of the new system through a linear combination given by:

$$
\begin{gathered}
\alpha_{\mathrm{M}}(t+h)=\sum_{i=1}^{6} p_{i}(t) \alpha_{i}(t)+p_{\mathrm{M}}(t) \alpha_{\mathrm{M}}(t), \\
\text { with } p_{\mathrm{M}}(t)+\sum_{i=1}^{6} p_{i}(t)=1
\end{gathered}
$$

Thereby, $\mathrm{M}$ refers to the mirror system with parameter $\alpha_{M}$. The mirror system itself participates at the 'competition' and is thus able to confirm its own parameter value $\alpha_{M}$ if it has a high switch-on probability $p_{\mathrm{M}}$.

Since it is neither practicable nor plausible in the sense of a 'natural' application to use the whole history, we estimate $p_{i}$ and $p_{\mathrm{M}}$, respectively, by means of a moving average over the recent past, say ten time steps, which corresponds to a time interval of length 0.1 . Fig. 3a shows the temporal behavior of the newly created parameter $\alpha_{M}$ compared with the constant parameter $\alpha_{E}$ of the external system. We observe a quick adaptation, however, with a relatively large remaining fluctuation between the neighbored parameter values. This fluctuation can be explained easily. A large change in the value of $\alpha_{M}$ unsynchronizes the mirror and external system for a while so that the neighbored systems have smaller control terms within that period, which in turn de-adjusts $\alpha_{\mathrm{M}}$. This can be avoided by limiting the changes of $\alpha_{\mathrm{M}}$. If one allows a maximum change of 0.0002 amplitude, this leads to a highly significant reduction of the fluctuation as can be seen in Fig. 3b.

In a previous paper (Diebner et al., 2001), we have shown that the adaptation works in a highly satisfactory manner even if the external Rössler system undergoes sudden changes. Remarkably, the adaptation is performed within roughly one cycle of the oscillator and it also works when two parameter values are unknown. Here, we proceed with the investigation of the adaptability with respect to the Lorenz system.

\section{Adaptation capability with respect to the Lorenz system}

We replace the pool of internal dynamics of the adaptive system given previously by a set of
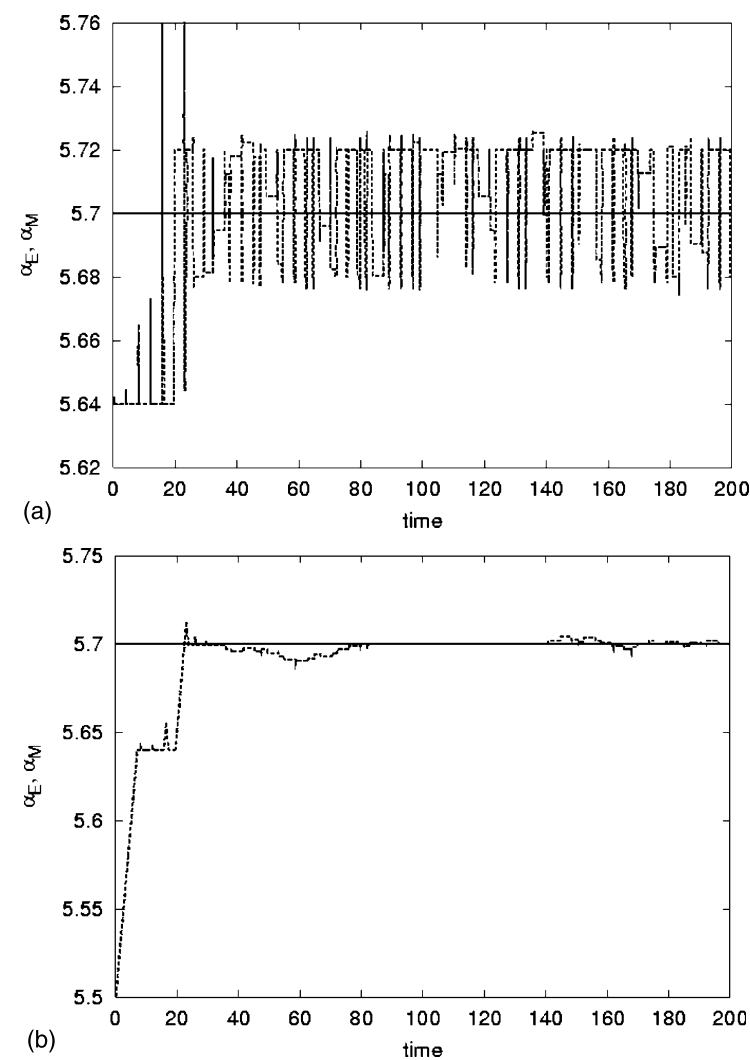

Fig. 3. (a) Parameter adaptation of the mirror system in the 'Rössler case' using Eq. (6) without further interference leads to an unsatisfactory fluctuation. (b) The fluctuation can be reduced very significantly by limiting the magnitudes of the parameter changes. 
Rössler systems according to Eqs. (5) and (4) by a set of Lorenz systems. We face the system with the $x$-variable of the externally given Lorenz system (Lorenz, 1963):

$\dot{x}_{\mathrm{E}}^{\mathrm{L}}=10\left(y_{\mathrm{E}}^{\mathrm{L}}-x_{\mathrm{E}}^{\mathrm{L}}\right)$

$\dot{y}_{\mathrm{E}}^{\mathrm{L}}=x_{\mathrm{E}}^{\mathrm{L}}\left(28-z_{\mathrm{E}}^{\mathrm{L}}\right)-y_{\mathrm{E}}^{\mathrm{L}}$

$\dot{z}_{\mathrm{E}}^{\mathrm{L}}=x_{\mathrm{E}}^{\mathrm{L}} y_{\mathrm{E}}^{\mathrm{L}}-\gamma_{\mathrm{E}} z_{\mathrm{E}}^{\mathrm{L}}$

where the parameter $\gamma_{\mathrm{E}}$ has been set to a value equal to 2.66, which has to be recognized and estimated by the adaptive system. The subscript $\mathrm{E}$ means 'external' and the superscript $\mathrm{L}$ stands for 'Lorenz'. The internal pool of six Lorenz systems is defined by the following set of parameters

$$
\begin{array}{ll}
\gamma_{1}=2.4, & \gamma_{2}=2.5, \\
\gamma_{5}=2.8, & \gamma_{6}=2.9,
\end{array}
$$

whereby the adaptation works analogously to Eq. (5). We create a mirror system exactly in the same manner as described above for the Rössler system. We start the mirror system with a parameter value of $\gamma_{M}=3.0$. The result for the parameter adaptation is depicted in Fig. 4a, whereby the temporary changes of the parameter have been limited to 0.0002 as in the above case. One sees a relatively large remaining fluctuation, which can be reduced significantly by choosing a finer limitation with an amplitude of $2 \times 10^{-5}$, which can be seen in Fig. $4 \mathrm{~b}$. This reduction of fluctuation leads to a relatively strong increase of adaptation time in the order of magnitude of 100 oscillator cycles.

The poorer adaptapility of Lorenz' system has to be discussed. First, one has to note that the dynamics behavior differs much less within the range of parameter values under investigation. Second, if the mirror system has a parameter value close to the external system, but not exactly identical, it may happen that the trajectory of one of the systems changes asynchronously to the other one, to the second 'wing' of the Lorenz attractor. This leads to a sudden increase of the control term and any other internal system that is momentarily on the same wing as the external one is preferred temporarily in the sense of the magnitude of the control term. Thus, if one counts the number of changes between the 'wings' as typical events until a sufficient adaptation takes place
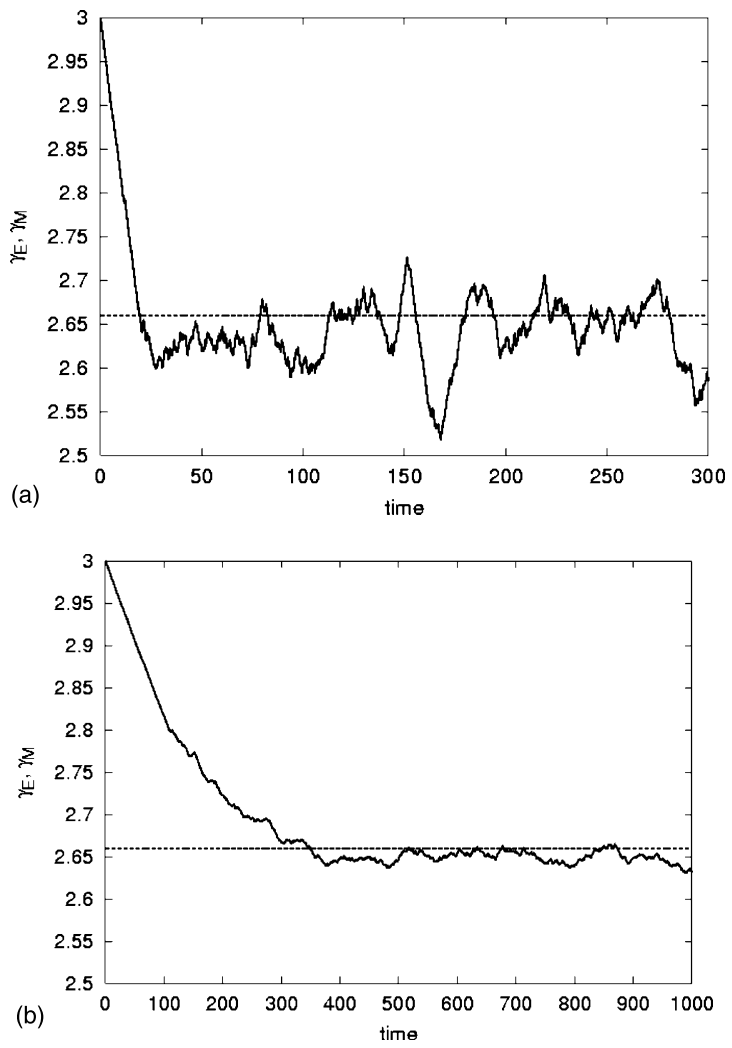

Fig. 4. (a) Parameter adaptation of the mirror system in the 'Lorenz case' with too weak limitation of the parameter changes. (b) A stronger limitation of the parameter changes enhances the precision of adaption on the cost of adaptation speed.

instead of counting the number of oscillator cycles as in the Rössler case we are again in the same range. In either case, we observe a convincing speed of adaptation.

With respect to brain dynamics, it is well known that nonlinear dynamics are more subtle to recognize. We need a longer experience (conditioning) of the dynamics to become familiar with it and to use it for predictions. Compared with the proposed adaptive system this means that the simulus needs longer adaptation time to handle chaotic dynamics. The adaptability behavior is a compromise between precision and speed of adaptation. Of course, the precision is also limited by the size of the internal pool. Anyhow, compared with existing methods for time series analyses of chaotic signals we are still on an innovative path. 


\section{Discussion}

The proposed system for dynamics recognition contains an element, called a mirror system, that mimics an external dynamics after parameter adaptation and is thus capable to simulate. Of course, if the external dynamics are already represented within the pool of internal dynamics, the adaptation is executed very quickly. Therefore, it would be advantageous to have a memory for frequently presented external dynamics. To introduce memory without exploding the dimensionality of the pool of internal dynamics, one can choose an existing element out of the pool that undergoes the adaptation, namely one that has not been used for a long time and at the same time is not too different from the external dynamics. Very much like in other natural processes, we thereby introduce memory with a certain loss rate.

A further task for future work is to extend the system to different families of dynamics. For example, one may add elements to the pool of internal dynamics that correspond to both the Lorenz as well as the Rössler system. There is almost no doubt that this system will recognize the correct dynamics if either a Rössler or a Lorenz dynamics is presented externally. It would be interesting to observe how the system reacts to a cross dynamics of Rössler plus Lorenz type. For example, if a sum signal:

$$
x(t)=\delta x_{1}^{\text {Rossler }}(t)+(1-\delta) x_{1}^{\text {Lorenz }}(t)
$$

is presented to the adaptive system, one can vary $\delta$ in the range between 0 and 1 to switch on 'Rössler' or 'Lorenz' or a cross type. This leads to the idea that for a flexible application one has to search for dynamical elements within the pool that allow for the creation of a large range of different dynamical types. The adaptation, therefore, has to be extended from pure parameter adaptation to recognition of basic dynamical elements that have to be combined. We are very confident that the proposed system has the potential to proceed in this direction. Note that it has been shown in a recent work (Meyer, 1995) that the generalized Rössler system in arbitrary many dimensions can be ruled back to a set of harmonic oscillators and one nonlinear element that have to be combined. This is indeed a first step towards a general decomposition of dynamics into basic modules.

To conclude, we expect a variety of technical and biomedical applications. Assume, for example, a patient suffering from chronical heart arrhythmias that urgently necessitate immediate therapeutical intervention when they appear suddenly. In this case, a rather well-working model dynamics exists (Holden and El Naschie, 1995) with which a pool of internal dynamics for an adaptive system can be created. This example may suffice to emphasize the potential relevance of the proposed real-time adaptive system.

Thank you, Michael.

\section{References}

Babloyantz, A., Lourenço, C., 1994. Computation with chaosa paradigm for cortical activity. Proc. Natl. Acad. Sci. USA 91, 9027-9031.

Conrad, M., 1983. Adaptability. Plenum, New York.

Diebner, H.H., Sahle, S., Hoff, A.A., 2001. A real-time adaptive system for dynamics recognition. Chaos, Sol. \& Frac. 13 (4), 781-786.

Hoff, A.A., 1994. Chaos control and neural classification. Z. Naturforsch. 49a, 589-593.

Hoff, A.A., 1997. Chaoskontrolle, Informationsverarbeitung und chemische Reaktionssysteme. Logos Verlag, Berlin.

Hoff, A.A., Diebner, H.H., Baier, G., 1995. Reversible control of chemical reaction systems. Z. Naturforsch. 50a, 11411146.

Holden, A.V., El Naschie, M.S., (Ed.), 1995. Nonlinear Phenomena in Excitable Physiological Systems. Chaos, Solitons \& Fractals, vol. 5, no. 3-4 (special issue).

Lorenz, E.N., 1963. Deterministic non-periodic flow. J. Atmos. Sci. 20, 130-141.

Lourenço, C., Babloyantz, A., 1994. Control of chaos in networks with delay: a model for synchronization of coritcal tissue. Neural Computation 6, 1141-1154.

Meyer, T., 1995. Das Verallgemeinerte Rössler System. Master's Thesis, University of Bayreuth, Lehrstuhl Experimentalphysik II.

Nicolis, J.S., Tsuda, I., 1999. Mathematical description of brain dynamics in perception and action. J. Consci. Studies $6,215-228$.

Prehn, H., 2001. The neuro-loop: biofeedback interfaces. In: Diebner, H.H., Druckrey, T., Weibel, P. (Eds.), Sciences of the Interface. Genista-Verlag, Tübingen.

Pyragas, K., 1992. Continuous control of chaos by self-controlling feedback. Phys. Lett. A 170, 421. 
Rössler, O.E., 1974. Adequate locomotion strategies for an abstract organism in an abstract environment - a relational approach to brain function. In: Lecture Notes in Biomathematics, vol. 4. Springer, Berlin.

Rössler, O.E., 1976. An equation for continuos chaos. Phys.
Lett. A 13, 397-398.

Skarda, C., Freeman, W., 1987. How brains make chaos in order to make sense of the world. Behav. Brain Sci. 10, 161-195. Tsuda, I., 2001. The plausibility of a chaotic brain theory. Behav. Brain Sci., in press. 